\title{
CURVATURE, DIAMETER, AND QUOTIENT MANIFOLDS
}

\author{
Burt Totaro
}

This paper gives improved counterexamples to a question by Grove ([11], 5.7). The question was whether for each positive integer $n$ and real number $D$, the simply connected closed Riemannian $n$-manifolds $M$ with sectional curvature $\geq-1$ and diameter $\leq D$ fall into only finitely many rational homotopy types. This was suggested by Gromov's theorem which bounds the Betti numbers of $M$ in terms of $n$ and $D$ [10]. It was known that there can be infinitely many integral homotopy types already in dimension 7, perhaps first by Aloff and Wallach [2]

Fang and Rong recently gave a negative answer to Grove's question in all dimensions $\geq 22$ ([7], Theorem B). We use certain biquotient manifolds, that is, quotients of homogeneous manifolds $G / H$ by a subgroup of $G$ which acts freely, to show that the question has a negative answer already in dimension 6 . Our examples are in fact nonnegatively curved. More precisely, we find infinitely many rational cohomology rings among simply connected closed Riemannian 6manifolds with nonnegative sectional curvature. (Of course, we can arrange that these manifolds also have diameter at most 1, by scaling.) The dimension 6 here is optimal, meaning that Grove's question has a positive answer in dimensions $\leq 5$. This follows from Gromov's bound on the Betti numbers, since the Betti numbers of a simply connected manifold of dimension $\leq 5$ determine its rational homotopy type up to finitely many possibilities. More precisely, the conjecture that simply connected manifolds of nonnegative curvature are integrally elliptic would imply, by Paternain and Petean ([15], Corollary 3.6), that simply connected 5-manifolds of nonnegative curvature fall into only 4 diffeomorphism classes: $S^{5}, S^{3} \times S^{2}$, the nontrivial $S^{3}$-bundle over $S^{2}$, and the $\mathrm{Wu}$ manifold $S U(3) / S O(3)[4]$.

Fang and Rong's examples have the merit of also having an upper bound on curvature. That is, for $n \geq 22$, Fang and Rong find numbers $C$ and $D$ such that there are infinitely many rational cohomology rings among simply connected closed Riemannian $n$-manifolds with curvature $-1 \leq K \leq C$ and diameter $\leq D$. The next main result of this paper is that such examples exist already among 7-manifolds. This is optimal, since Fang and Rong [6], and also Tuschmann [19], have proved that in dimensions $\leq 6$ there are only finitely many diffeomorphism classes in the given class of manifolds. Finally, in dimension 9, we use biquotients to give a similar counterexample using only nonnegatively curved manifolds. That is, for some $C$ and $D$, there are infinitely many rational

Received July 23, 2002. 
cohomology rings among simply connected closed 9-manifolds with curvature $0 \leq K \leq C$ and diameter $\leq D$.

To conclude, one can ask what substitute for Grove's question might be true. For the problem with an upper curvature bound, there is already a remarkable substitute for Grove's question, the Petrunin-Tuschmann theorem ([16], Corollary 0.2). Namely, for each $n, C$, and $D$, there is a finite set of closed smooth manifolds $E_{i}$ of dimension $\geq n$ such that any simply connected closed Riemannian $n$-manifold with curvature $-1 \leq K \leq C$ and diameter $\leq D$ is diffeomorphic to the quotient of some $E_{i}$ by a free action of a torus. Thus, in Fang and Rong's examples and in our examples in dimensions 7 and 9, the infiniteness comes entirely from considering quotients of a single manifold by different free torus actions.

In section 4, we suggest some possible substitutes for Grove's question with no upper curvature bound.

\section{Counterexamples to Grove's question among nonnegatively curved 6-manifolds}

Here we prove:

Theorem 1.1. There are infinitely many isomorphism classes of rational cohomology rings among simply connected closed Riemannian 6-manifolds with nonnegative sectional curvature.

As explained in the introduction, this gives a negative answer to Grove's question in dimension 6, which is optimal. Also, it follows from the theorem of Tuschmann [19] and Fang-Rong [6] discussed in the introduction that there cannot be an upper bound on the curvature of the manifolds we construct, if we fix their diameter to be 1.

Proof. The 6-manifolds $M$ we construct will all be biquotients, of the form $\left(S^{3}\right)^{3} /\left(S^{1}\right)^{3}$ for different free isometric actions of the group $\left(S^{1}\right)^{3}$ on the Riemannian manifold $\left(S^{3}\right)^{3}$. Like all biquotients, these manifolds $M$ have nonnegative sectional curvature, by O'Neill's curvature formula for Riemannian submersions [14], which we state in the proof of Theorem 2.1.

Let $\left(S^{1}\right)^{2} \subset S O(4)$ be the standard maximal torus, with $(\lambda, \mu) \in\left(S^{1}\right)^{2}$ acting isometrically on $S^{3} \subset \mathbf{C}^{2}$ by $(\lambda, \mu)(u, v)=(\lambda u, \mu v)$. Therefore we have a natural isometric action of $\left(S^{1}\right)^{6}$ on $\left(S^{3}\right)^{3}$. The actions of $\left(S^{1}\right)^{3}$ on $\left(S^{3}\right)^{3}$ we consider will be given by homomorphisms $\left(S^{1}\right)^{3} \rightarrow\left(S^{1}\right)^{6}$, which we will specify further as we go along. As a first simplification, let us assume that the homomorphism $\left(S^{1}\right)^{3} \rightarrow\left(S^{1}\right)^{6} \rightarrow\left(S^{1}\right)^{3}$ which gives the action of $\left(S^{1}\right)^{3}$ on the coordinates $\left(u_{1}, u_{2}, u_{3}\right)$ is the identity. Given this, the homomorphisms $\left(S^{1}\right)^{3} \rightarrow\left(S^{1}\right)^{6}$ we consider will be determined by a $3 \times 3$ matrix of integers

$$
\left(\begin{array}{lll}
a_{1} & a_{2} & a_{3} \\
b_{1} & b_{2} & b_{3} \\
c_{1} & c_{2} & c_{3}
\end{array}\right),
$$


with the action of $\left(S^{1}\right)^{3}$ on $\left(S^{3}\right)^{3}$ given by

$$
\begin{aligned}
& \left(\lambda_{1}, \lambda_{2}, \lambda_{3}\right)\left(\left(u_{1}, v_{1}\right),\left(u_{2}, v_{2}\right),\left(u_{3}, v_{3}\right)\right) \\
& =\left(\left(\lambda_{1} u_{1}, \lambda_{1}^{a_{1}} \lambda_{2}^{a_{2}} \lambda_{3}^{a_{3}} v_{1}\right),\left(\lambda_{2} u_{2}, \lambda_{1}^{b_{1}} \lambda_{2}^{b_{2}} \lambda_{3}^{b_{3}} v_{2}\right),\left(\lambda_{3} u_{3}, \lambda_{1}^{c_{1}} \lambda_{2}^{c_{2}} \lambda_{3}^{c_{3}} v_{3}\right)\right) .
\end{aligned}
$$

To check whether this action of $\left(S^{1}\right)^{3}$ on $\left(S^{3}\right)^{3}$ is free, one sees easily that it suffices to check freeness at the 8 points $\left(p_{1}, p_{2}, p_{3}\right)$ in $\left(S^{3}\right)^{3}$ with each $p_{i}$ equal to $(1,0)$ or $(0,1)$. Here freeness at the point $((1,0),(1,0),(1,0))$ is automatic by our choice of the action on the variables $u_{i}$. Freeness at the 3 points with one $(0,1)$ component means that the diagonal entries $a_{1}, b_{2}, c_{3}$ of our matrix are \pm 1 . Freeness at the 3 points with two $(0,1)$ component means that the 3 determinants

$$
\operatorname{det}\left(\begin{array}{ll}
b_{2} & b_{3} \\
c_{2} & c_{3}
\end{array}\right), \operatorname{det}\left(\begin{array}{ll}
a_{1} & a_{3} \\
c_{1} & c_{3}
\end{array}\right), \operatorname{det}\left(\begin{array}{ll}
a_{1} & a_{2} \\
b_{1} & b_{2}
\end{array}\right)
$$

are \pm 1 . Finally, freeness at the point $((0,1),(0,1),(0,1))$ means that the whole $3 \times 3$ matrix has determinant \pm 1 .

Let us choose our $3 \times 3$ integer matrix to have the form

$$
\left(\begin{array}{ccc}
1 & 0 & 0 \\
b_{1} & 1 & 1 \\
c_{1} & 2 & 1
\end{array}\right) .
$$

Then the above conditions are satisfied for all integers $b_{1}$ and $c_{1}$. Thus the corresponding actions of $\left(S^{1}\right)^{3}$ on $\left(S^{3}\right)^{3}$ are free. We will show that the corresponding quotient manifolds $M=\left(S^{3}\right)^{3} /\left(S^{1}\right)^{3}$ have infinitely many non-isomorphic rational cohomology rings.

To compute the cohomology ring of such a manifold $M$, we consider the associated fibration

$$
\left(S^{3}\right)^{3} \rightarrow\left(S^{3}\right)^{3} /\left(S^{1}\right)^{3} \rightarrow\left(B S^{1}\right)^{3} .
$$

Thus we can consider $M$, up to homotopy, as being obtained from $\left(B S^{1}\right)^{3}$ by passing three times from the base space to the total space of an $S^{3}$-bundle. The cohomology ring of $\left(B S^{1}\right)^{3}=\left(\mathbf{C P}^{\infty}\right)^{3}$ is the polynomial ring $\mathbf{Z}\left[x_{1}, x_{2}, x_{3}\right]$. By our choice of the action of $\left(S^{1}\right)^{3}$ on $\left(S^{3}\right)^{3}$, our three $S^{3}$-bundles over $\left(B S^{1}\right)^{3}$ have Euler classes in $H^{4}\left(\left(B S^{1}\right)^{3}, \mathbf{Z}\right)$ of the form $x_{1}^{2}, x_{2}\left(b_{1} x_{1}+x_{2}+x_{3}\right)$, and $x_{3}\left(c_{1} x_{1}+2 x_{2}+x_{3}\right)$. We observe that these elements form a regular sequence in the polynomial ring $\mathbf{Z}\left[x_{1}, x_{2}, x_{3}\right]$. Therefore, applying the spectral sequence successively for these three $S^{3}$-bundles shows that

$$
H^{*}(M, \mathbf{Z})=\mathbf{Z}\left[x_{1}, x_{2}, x_{3}\right] /\left(x_{1}^{2}, x_{2}\left(b_{1} x_{1}+x_{2}+x_{3}\right), x_{3}\left(c_{1} x_{1}+2 x_{2}+x_{3}\right)\right) .
$$

It remains to show that these 6-manifolds $M$ have infinitely many nonisomorphic rational cohomology rings, as the integers $b_{1}$ and $c_{1}$ vary. It turns out that later calculations are slightly simplified if we define the rational numbers $a=c_{1} / 4$ and $b=\left(2 b_{1}-c_{1}\right) / 4$, so that $b_{1}=2(a+b)$ and $c_{1}=4 a$. Things 
will also simplify if we multiply the second relation by 2 . In these terms, $M$ has rational cohomology ring

$H^{*}(M, \mathbf{Q})=\mathbf{Q}\left[x_{1}, x_{2}, x_{3}\right] /\left(x_{1}^{2}, 2 x_{2}\left(2(a+b) x_{1}+x_{2}+x_{3}\right), x_{3}\left(4 a x_{1}+2 x_{2}+x_{3}\right)\right)$.

We will only consider the manifolds $M$ associated to integers $b_{1}$ and $c_{1}$ which are not both zero. Then $a$ and $b$ are not both zero.

It turns out that we only need to consider the rational cohomology ring of $M$ in degrees $\leq 4$. In this range, the ring is described by a 3-dimensional vector space $V=H^{2}(M, \mathbf{Q})$ together with a 3 -dimensional linear subspace of $S^{2} V$, the kernel of the product map $S^{2} V \rightarrow H^{4}(M, \mathbf{Q})$. We need to extract a more understandable invariant from this 3-dimensional linear system of quadrics, which is spanned by the above 3 relations. One approach, which I learned from Wall [21], is to consider the determinant of the quadratic form given by an arbitrary linear combination of the above 3 relations,

$$
\lambda x_{1}^{2}+\mu \cdot 2 x_{2}\left(2(a+b) x_{1}+x_{2}+x_{3}\right)+\nu x_{3}\left(4 a x_{1}+2 x_{2}+x_{3}\right) .
$$

The determinant of this quadratic form is:

$$
\operatorname{det}\left(\begin{array}{ccc}
\lambda & 2(a+b) \mu & 2 a \nu \\
2(a+b) \mu & 2 \mu & \mu+\nu \\
2 a \nu & \mu+\nu & \nu
\end{array}\right)=-\lambda \mu^{2}-\lambda \nu^{2}+4\left(a^{2}-b^{2}\right) \mu^{2} \nu+8 a b \mu \nu^{2} .
$$

This is a more geometrically understandable object, a cubic curve over $\mathbf{Q}$ which is an invariant of the rational cohomology ring of $M$, modulo the action of $G L(3, \mathbf{Q})$ on $\lambda, \mu, \nu$ and modulo scalars. For convenience, write $\alpha=4\left(a^{2}-b^{2}\right)$ and $\beta=8 a b$, so the cubic has the form

$$
-\lambda \mu^{2}-\lambda \nu^{2}+\alpha \mu^{2} \nu+\beta \mu \nu^{2}=0
$$

Since $a$ and $b$ are not both zero, $\alpha$ and $\beta$ are not both zero. Then we compute that the cubic curve has exactly one singular point, a node at the point $[1,0,0]$ in $\mathbf{P}^{2}$.

We still need to extract a more computable invariant from this nodal cubic over $\mathbf{Q}$. We use that this curve has 3 inflection points over the algebraic closure of $\mathbf{Q}$, apart from the singular point. The lines from these 3 inflection points to the singular point $[1,0,0]$ are specified by the point $[\mu, \nu] \in \mathbf{P}^{1}$ associated to each inflection point $[\lambda, \mu, \nu] \in \mathbf{P}^{2}$. Here we are thinking of $\mathbf{P}^{1}$ as the space of lines through the singular point $[1,0,0]$ in $\mathbf{P}^{2}$. Computing shows that lines through the 3 inflection points are the 3 roots of the equation:

$$
\beta \mu^{3}-3 \alpha \mu^{2} \nu-3 \beta \mu \nu^{2}+\alpha \nu^{3}=0 .
$$

The two tangent lines to the nodal cubic curve at its singular point are described by the binary quadratic form $\mu^{2}+\nu^{2}$. Therefore, knowing the nodal cubic curve modulo scalars and automorphisms of $\mathbf{P}^{2}$ determines the above binary cubic form modulo scalars and modulo automorphisms of $\mathbf{P}^{1}$ which preserve the binary quadratic form $\mu^{2}+\nu^{2}$ up to scalars. This automorphism group is an orthogonal group $O(2)$ times the scalars, and so it has two connected components. 
The identity component consists of the automorphisms

$$
\begin{aligned}
& \mu \mapsto c \mu+d \nu \\
& \nu \mapsto-d \mu+c \nu
\end{aligned}
$$

with $c, d \in \mathbf{Q}$, not both zero. We compute that this group acts on the above binary cubic by the following change in $\alpha$ and $\beta$ :

$$
\alpha+\beta i \mapsto(\alpha+\beta i)(c+d i)^{3},
$$

where $i=\sqrt{-1}$ as usual. All our notation has been chosen in order to make this formula as simple as possible.

Thus, to the above binary cubic we associate the number $\alpha+\beta i$ in $K=\mathbf{Q}(i)$. It is nonzero since we assumed $\alpha$ and $\beta$ are not both 0 . Scaling the binary cubic leaves a well-defined class in $K^{*} / \mathbf{Q}^{*}$. Making the above coordinate change leaves a well-defined class in $\left.K^{*} /\left(\mathbf{Q}^{*} \cdot\left(K^{*}\right)^{3}\right)\right)=\left(K^{*} / 3\right) /\left(\mathbf{Q}^{*} / 3\right)$. Finally, we have to consider the effect of a coordinate change not in the identity component of the above orthogonal group; for example, we can switch $\mu$ and $\nu$. This changes $\alpha+\beta i$ to $\beta+\alpha i$. To sum up, we can say that the rational cohomology ring of the 6-manifold $M$ determines an unordered pair of elements of the group $\left(K^{*} / 3\right) /\left(\mathbf{Q}^{*} / 3\right)$, the class of $\alpha+\beta i$ and the class of $\beta+\alpha i$.

We can now see that we have infinitely many isomorphism classes of rational cohomology rings among the 6-manifolds $M$. First, the group $\left(K^{*} / 3\right) /\left(\mathbf{Q}^{*} / 3\right)$ is infinite. This follows, for example, from the existence of infinitely many prime numbers $p$ which split in $K=\mathbf{Q}(i)$, namely all primes $p \equiv 1(\bmod 4)$. If $\pi_{1}$ and $\pi_{2}$ are the two prime ideals in $K$ which lie over $p$, then $\operatorname{ord}_{\pi_{1}}(x)-\operatorname{ord}_{\pi_{2}}(x)$ is a surjective homomorphism from $\left(K^{*} / 3\right) /\left(\mathbf{Q}^{*} / 3\right)$ to $\mathbf{Z} / 3$. Since we have infinitely many such homomorphisms, only finitely many of which can be nontrivial on a given element of $K^{*}$, the group $\left(K^{*} / 3\right) /\left(\mathbf{Q}^{*} / 3\right)$ is infinite.

Furthermore, any element of the group $\left(K^{*} / 3\right) /\left(\mathbf{Q}^{*} / 3\right)$ has the form $\alpha+\beta i$ for some $\alpha, \beta$ coming from a 6 -manifold $M$ as above. Indeed, the 6 -manifold $M$ is described by a pair of integers $b_{1}, c_{1}$, and then our definitions say that

$$
\begin{aligned}
a & =c_{1} / 4 \\
b & =\left(2 b_{1}-c_{1}\right) / 4 \\
\alpha+\beta i & =4(a+b i)^{2} .
\end{aligned}
$$

Since the group $\left(K^{*} / 3\right) /\left(\mathbf{Q}^{*} / 3\right)$ is 3 -torsion, every element $\alpha+\beta i$ is a square in this group, and so every element can be written as $4(a+b i)^{2}$ for some $a, b \in \mathbf{Q}$. Also, multiplying $a+b i$ by any nonzero integer does not change its class in $\left(K^{*} / 3\right) /\left(\mathbf{Q}^{*} / 3\right)$, so every element of the latter group has the form $4(a+b i)^{2}$ for some $a, b \in \mathbf{Z}$. Then the corresponding numbers $b_{1}$ and $c_{1}$ are also integers. Thus we have shown that the 6 -manifolds we consider can give rise to any element of the infinite group $\left(K^{*} / 3\right) /\left(\mathbf{Q}^{*} / 3\right)$.

To be precise, the invariant of the rational cohomology ring we defined is an unordered pair of elements of this group. This is enough to show that there are 
infinitely many isomorphism clases of rational cohomology rings among these 6-manifolds.

\section{Counterexamples to Grove's question among 7-manifolds with upper curvature bound}

Here we prove:

Theorem 2.1. There are numbers $C$ and $D$ such that there are infinitely many isomorphism classes of rational cohomology rings among simply connected closed Riemannian 7-manifolds with curvature $-1 \leq K \leq C$ and diameter $\leq D$.

As mentioned in the introduction, this strengthens the examples of Fang and Rong ([7], Theorem B), by lowering the dimension from 22 to 7 . The dimension 7 is optimal, by the theorem of Tuschmann [19] and Fang-Rong [6], as discussed in the introduction.

Proof. The manifolds we construct will all be quotients of a fixed manifold $E$ by different free torus actions. In fact, this is the only way to get examples as in the theorem, by the Petrunin-Tuschmann theorem ([16], 0.2), as discussed in the introduction.

In this case, $E$ will be an 11-manifold with a free $\left(S^{1}\right)^{5}$-action. Before defining $E$, we will construct a 6 -manifold $M$ which will be the quotient $E /\left(S^{1}\right)^{5}$. By Sullivan [18], Theorem 13.2, for any graded-commutative $\mathbf{Q}$-algebra which is $\mathbf{Q}$ in degree 0, 0 in degree 1, and which satisfies Poincaré duality of dimension 6 , there is a smooth simply connected 6 -manifold $M$ with the given rational cohomology ring. (One could also use Wall's more precise results on 6-manifolds [20].) We will take $M$ to have $b_{2}=5$ and $b_{3}=0$. Let $x_{0}, \ldots, x_{4}$ be a basis for $V:=H^{2}(M, \mathbf{Q})$. Then we choose $M$ so that the cubic form on $H^{2}(M, \mathbf{Q})$ is given by

$$
\int_{M}\left(a_{0} x_{0}+\ldots+a_{4} x_{4}\right)^{3}=c\left(a_{0}^{2} a_{1}+a_{1}^{2} a_{2}+a_{2}^{2} a_{3}+a_{3}^{2} a_{4}+a_{4}^{2} a_{0}\right)
$$

for some nonzero constant $c$. The corresponding cubic 3 -fold in $\mathbf{P}^{4}$ is known as the Klein cubic. It is not the only cubic form one could use, but calculations with it are particularly easy. For example, Adler used the Klein cubic as a tool to describe the Hessian quintic 3-fold of a general cubic 3-fold ([1], Appendix IV). Using the above formula for the cubic form, it is easy to check that the product map $S^{2} H^{2}(M, \mathbf{Q}) \rightarrow H^{4}(M, \mathbf{Q})$ is surjective.

Since $M$ is simply connected, $H^{2}(M, \mathbf{Z})$ is torsion-free and hence isomorphic to $\mathbf{Z}^{5}$, since $H^{2}(M, \mathbf{Q})$ is the 5-dimensional vector space $V$. Let $E$ be the total space of the corresponding $\left(S^{1}\right)^{5}$-bundle over $M$. Thus $E$ is an 11-manifold with a free $\left(S^{1}\right)^{5}$-action. Choose a Riemannian metric on $E$ preserved by the torus action. We can scale the metric so as to have curvature $\geq-1$. Let $D$ be the diameter of $E$.

We consider the 7-manifolds $Y$ which are quotients of $E$ by subtori $\left(S^{1}\right)^{4} \subset$ $\left(S^{1}\right)^{5}$. These 7 -manifolds are all simply connected. With the metric induced 
from $E$, they all have diameter $\leq D$. By O'Neill's formula [14], sectional curvature increases under Riemannian submersions, and so all these quotient manifolds have curvature $\geq-1$. For clarity, we recall O'Neill's formula here. Let $\pi: E \rightarrow M$ be a Riemannian submersion. Let $X, Y$ be linearly independent vector fields on an open subset of $E$ which are orthogonal to the fibers of $\pi$ ("horizontal" vector fields). Let $K$ denote sectional curvature on $E$ and $M$. Then

$$
K\left(\pi_{*} X, \pi_{*} Y\right)=K(X, Y)+3\left|\left(\nabla_{X} Y\right)_{v}\right|^{2} /|X \wedge Y|^{2},
$$

where the subscript $v$ denotes the projection to the tangent bundle of the fibers of $\pi$ ("vertical" projection).

Because $\left(S^{1}\right)^{5}$ acts freely on $E$, there is also an upper bound $C$ for the sectional curvature of all quotients of $E$ by subtori $\left(S^{1}\right)^{4} \subset\left(S^{1}\right)^{5}$. The idea here goes back to Eschenburg [5], Proposition 22. Namely, O'Neill's formula shows that the sectional curvature of a quotient manifold $E /\left(S^{1}\right)^{4}$ can be computed locally on $E$. Furthermore, the same formula for the curvature formally makes sense for the non-closed subgroup of $\left(S^{1}\right)^{5}$ associated to any real linear subspace $\mathbf{R}^{4}$ in the Lie algebra $\mathbf{R}^{5}$ of $\left(S^{1}\right)^{5}$, using that $\left(S^{1}\right)^{5}$ acts freely on $E$. The "curvature" so defined is continuous on the compact manifold of all subspaces $\mathbf{R}^{4} \subset \mathbf{R}^{5}$ and all 2-planes in the tangent bundle of $E$ which are orthogonal to the associated foliation of $E$. Therefore, there is a uniform upper bound for this curvature function, and hence for the curvature of all quotients $E /\left(S^{1}\right)^{4}$ associated to subtori $\left(S^{1}\right)^{4} \subset\left(S^{1}\right)^{5}$.

It remains to show that the different 7 -manifolds $Y$ have infinitely many nonisomorphic rational cohomology rings. We will only consider the cohomology ring in degrees $\leq 4$. Here $Y$ is an $S^{1}$-bundle over the 6 -manifold $M$. We use the corresponding spectral sequence to compute the rational cohomology of $Y$. First, $W:=H^{2}(Y, \mathbf{Q})=H^{2}\left(\left(B S^{1}\right)^{4}, \mathbf{Q}\right)$ is the 4-dimensional space $V /(\mathbf{Q} \cdot y)$, where $y$ in $V=H^{2}\left(\left(B S^{1}\right)^{5}, \mathbf{Q}\right)$ is dual to the subtorus $\left(S^{1}\right)^{4} \subset\left(S^{1}\right)^{5}$ used to define $Y$. Also, by the same spectral sequence, the image of $S^{2} W$ in $H^{4}(Y, \mathbf{Q})$ is $H^{4}(M, \mathbf{Q}) /(y \cdot V)$.

It seems that "general" $S^{1}$-bundles $Y$ over $M$ will not have interesting cohomology rings. Fortunately, we can exhibit a special class of $S^{1}$-bundles which do have interesting cohomology rings. Namely, consider $S^{1}$-bundles $Y$ over $M$ corresponding to elements $y=a_{0} x_{0}-a_{1} x_{1}+\left(a_{1}^{3} / a_{0}^{2}\right) x_{3}$ in $V=H^{2}(M, \mathbf{Q})$, for nonzero rational numbers $a_{0}$ and $a_{1}$. (For each nonzero element $y$ up to scalars in $H^{2}(M, \mathbf{Q})$, there is a corresponding $S^{1}$-bundle $Y$ over $M$ which is simply connected.) That is, $y$ is a rational point on a certain cuspidal cubic curve in the projective space $\mathbf{P}^{4}$ of lines in $V$. We will see that the corresponding $S^{1}$-bundles $Y$ over $M$ have infinitely many non-isomorphic rational cohomology rings.

The first useful property of points $y$ as above is that multiplication by $y$, from $V=H^{2}(M, \mathbf{Q})$ to $H^{4}(M, \mathbf{Q}) \cong V^{*}$, is not an isomorphism, as we compute directly from the cubic form. In the 19th-century terminology, which we will not really need, this means that the above cuspidal curve lies on the Hessian quintic 3 -fold of the given cubic 3-fold. In fact, we compute that the kernel of 
multiplication by $y$, from $H^{2} M$ to $H^{4} M$, is 1-dimensional, spanned by

$$
z:=a_{0} x_{0}+a_{1} x_{1}+\left(a_{0}^{2} / a_{1}\right) x_{2} .
$$

I have the impression that what makes the following proof work is the curious fact that the birational involution of the Hessian quintic which takes $y$ to $z$ transforms the cuspidal cubic curve of points $y$ to the smooth conic curve of points $z$. In general, it is well known that a quintic 3-fold will have many rational curves, but the difference between these two curves still seems surprising. In any case, the following proof will not use these vague ideas.

Because multiplication by $y$ from $H^{2} M$ to $H^{4} M$ has 1-dimensional kernel, the cokernel $H^{4} M /\left(y \cdot H^{2} M\right)$ is also 1-dimensional. We identified this cokernel with the image of the product map $S^{2}\left(H^{2} Y\right) \rightarrow H^{4} Y$. Thus the cup product on the 7-manifold $Y$ determines a nonzero quadratic form on $H^{2}(Y, \mathbf{Q})$, well-defined up to scalars. Explicitly, using that $y z=0 \in H^{4} M$, this quadratic form is defined, up to scalars, by

$$
(u, v):=\int_{M} u v z
$$

for $u, v \in H^{2} M /(\mathbf{Q} \cdot y) \cong H^{2} Y$.

Since $H^{2}(Y, \mathbf{Q})$ has dimension 4 , which is even, the determinant in $\mathbf{Q} /\left(\mathbf{Q}^{*}\right)^{2}$ of a quadratic form on $H^{2}(Y, \mathbf{Q})$ is not changed upon multiplying the quadratic form by a nonzero scalar. Thus the determinant of the above quadratic form in $\mathbf{Q} /\left(\mathbf{Q}^{*}\right)^{2}$ is an invariant of the rational cohomology ring of $Y$.

Let $a_{2}=a_{0}^{2} / a_{1}$, so that $z=a_{0} x_{0}+a_{1} x_{1}+a_{2} x_{2}$. We compute that the quadratic form $(u, v):=\int_{M} u v z$ on the 5-dimensional space $H^{2} M$ is given, up to a constant factor, by the matrix

$$
\left(\begin{array}{ccccc}
a_{1} & a_{0} & 0 & 0 & 0 \\
a_{0} & a_{2} & a_{1} & 0 & 0 \\
0 & a_{1} & 0 & a_{2} & 0 \\
0 & 0 & a_{2} & 0 & 0 \\
0 & 0 & 0 & 0 & a_{0}
\end{array}\right) .
$$

This matrix has determinant 0 , because this quadratic form on $H^{2} M$ has at least a 1-dimensional kernel spanned by $y$. To compute the determinant of the resulting quadratic form on $H^{2} M /(\mathbf{Q} \cdot y) \cong H^{2} Y$, we can use the lower right $4 \times 4$ minor, which has determinant equal to:

$$
-a_{2}^{3} a_{0}=-\left(a_{0}^{2} / a_{1}\right)^{3} a_{0}=-a_{0}^{7} / a_{1}^{3} \sim-a_{0} / a_{1} \in \mathbf{Q}^{*} /\left(\mathbf{Q}^{*}\right)^{2} .
$$

Thus, as we vary the nonzero rational numbers $a_{0}$ and $a_{1}$, the determinant of the quadratic form up to scalars on $H^{2} Y$ can take arbitrary values in the infinite group $\mathbf{Q}^{*} /\left(\mathbf{Q}^{*}\right)^{2}$. It follows that the 7-manifolds $Y$ have infinitely many non-isomorphic rational cohomology rings. 


\section{Counterexamples to Grove's question among nonnegatively curved 9-manifolds with upper curvature bound}

Here we prove:

Theorem 3.1. There are numbers $C$ and $D$ such that there are infinitely many isomorphism classes of rational cohomology rings among simply connected closed Riemannian 9-manifolds with curvature $0 \leq K \leq C$ and diameter $\leq D$.

It seems an interesting challenge to find out whether the dimension here can be improved. Another comment is that, at least in slightly higher dimensions, there are examples as in Theorem 3.1 which have infinitely many isomorphism classes of cohomology rings with complex coefficients.

Proof. The manifolds we construct will all be quotients of a fixed manifold by different free torus actions, as in Theorem 2.1. In fact, this is the only way to get examples as in the theorem, by the Petrunin-Tuschmann theorem ([16], 0.2), as discussed in the introduction.

Precisely, we consider biquotients of the form $\left(S^{3}\right)^{4} /\left(S^{1}\right)^{3}$, for the different subgroups $\left(S^{1}\right)^{3} \subset\left(S^{1}\right)^{4}$, where we will specify a free isometric action of $\left(S^{1}\right)^{4}$ on $\left(S^{3}\right)^{4}$, not the obvious one. These 9 -manifolds are all simply connected. With the metric induced from the standard metric on $\left(S^{3}\right)^{4}$, they all have diameter $\leq D$ where $D$ is the diameter of $\left(S^{3}\right)^{4}$. By O'Neill's formula [14], sectional curvature increases under Riemannian submersions, and so all these quotient manifolds have nonnegative curvature. Finally, by the same argument as in the proof of Theorem 2.1, since the whole group $\left(S^{1}\right)^{4}$ acts freely on $\left(S^{3}\right)^{4}$, there is also an upper bound $C$ for the sectional curvature of all quotients of $\left(S^{3}\right)^{4}$ by subtori $\left(S^{1}\right)^{3} \subset\left(S^{1}\right)^{4}$.

We now explain the free isometric action of $\left(S^{1}\right)^{4}$ on $\left(S^{3}\right)^{4}$ which we will use. We think of $S^{3}$ as the unit sphere in $\mathbf{C}^{2}$. For any $4 \times 4$ lower-triangular matrix $A=\left(a_{i j}\right)$ of integers with 1's on the diagonal, the following isometric action of $\left(S^{1}\right)^{4}$ on $\left(S^{3}\right)^{4}$ is free:

$$
\begin{aligned}
\left(\lambda_{1}, \lambda_{2}, \lambda_{3}, \lambda_{4}\right)\left(\left(u_{1}, v_{1}\right),\left(u_{2}, v_{2}\right),\left(u_{3}, v_{3}\right),\left(u_{4}, v_{4}\right)\right) & \\
= & \left(\left(\lambda_{1} u_{1},\left(\prod_{j} \lambda_{j}^{a_{1 j}}\right) v_{1}\right), \ldots,\left(\lambda_{4} u_{4},\left(\prod_{j} \lambda_{j}^{a_{4 j}}\right) v_{4}\right)\right) .
\end{aligned}
$$

Let $M$ be the quotient 8 -manifold $\left(S^{3}\right)^{4} /\left(S^{1}\right)^{4}$. By viewing $M$ as the total space of a 4 -fold iterated $S^{3}$-bundle over $\left(B S^{1}\right)^{4}$, we find that $M$ has cohomology ring

$$
\begin{array}{r}
H^{*}(M, \mathbf{Z})=\mathbf{Z}\left[x_{1}, x_{2}, x_{3}, x_{4}\right] /\left(x_{1}^{2}, x_{2}\left(a_{21} x_{1}+x_{2}\right), x_{3}\left(a_{31} x_{1}+a_{32} x_{2}+x_{3}\right),\right. \\
\left.x_{4}\left(a_{41} x_{1}+a_{42} x_{2}+a_{43} x_{3}+x_{4}\right)\right) .
\end{array}
$$

We now specialize the integers $a_{i j}$ to make the cohomology ring of the 8manifold $M$ equal to:

$$
H^{*}(M, \mathbf{Z})=\mathbf{Z}\left[x_{1}, x_{2}, x_{3}, x_{4}\right] /\left(x_{1}^{2}, x_{2}^{2}, x_{3}\left(x_{1}+2 x_{2}+x_{3}\right), x_{4}\left(x_{1}+2 x_{2}+x_{4}\right)\right) .
$$


Changing variables over $\mathbf{Q}$ by $x_{3} \mapsto x_{3}-x_{1} / 2-x_{2}$ and $x_{4} \mapsto x_{4}-x_{1} / 2-x_{2}$, we find that

$$
H^{*}(M, \mathbf{Q})=\mathbf{Q}\left[x_{1}, x_{2}, x_{3}, x_{4}\right] /\left(x_{1}^{2}, x_{2}^{2}, x_{3}^{2}-x_{1} x_{2}, x_{4}^{2}-x_{1} x_{2}\right) .
$$

We want to show that the 9 -manifolds $Y=\left(S^{3}\right)^{4} /\left(S^{1}\right)^{3}$ associated to subtori $\left(S^{1}\right)^{3} \subset\left(S^{1}\right)^{4}$ can have infinitely many non-isomorphic rational cohomology rings. As in our previous examples, we only need to consider the cohomology ring of $Y$ in degrees $\leq 4$. Here $Y$ can be the $S^{1}$-bundle over $M$ corresponding to any element of $H^{2}(M, \mathbf{Z})$ which generates a summand of $H^{2}(M, \mathbf{Z})$. In particular, for each $a, b, c \in \mathbf{Q}$, there is an $S^{1}$-bundle $Y$ over $M$ such that $H^{2}(Y, \mathbf{Q})$ is the quotient of $H^{2}(X, \mathbf{Q})$ by the line spanned by $x_{4}-\left(a x_{1}+b x_{2}+c x_{3}\right)$. Then $H^{2}(Y, \mathbf{Q})$ is spanned by $x_{1}, x_{2}, x_{3}$. We compute using the spectral sequence of this $S^{1}$-bundle that the kernel of the cup product $S^{2} H^{2}(Y, \mathbf{Q}) \rightarrow H^{4}(Y, \mathbf{Q})$ is the linear system of quadrics spanned by

$$
x_{1}^{2}, x_{2}^{2}, x_{3}^{2}-x_{1} x_{2},\left(a x_{1}+b x_{2}+c x_{3}\right)^{2}-x_{1} x_{2} .
$$

To prove the theorem, it suffices to show that we obtain infinitely many linear systems of quadrics modulo coordinate changes in $G L(3, \mathbf{Q})$, as $a, b, c \in \mathbf{Q}$ vary. Let us assume that $a, b, c$ are all nonzero, as we are free to.

The idea is to consider what squares of linear forms in $x_{1}, x_{2}, x_{3}$ belong to this linear system, over a given field $k$ containing $\mathbf{Q}$. It is easy to check that $x_{1}^{2}$ and $x_{2}^{2}$ are the only squares of linear forms, up to scalars, in the span of $x_{1}^{2}$, $x_{2}^{2}$, and $x_{3}^{2}-x_{1} x_{2}$. So suppose that we have a linear form with coefficients in $k$ whose square is a nonzero multiple of $\left(a x_{1}+b x_{2}+c x_{3}\right)^{2}-x_{1} x_{2}$ plus a linear combination of $x_{1}^{2}, x_{2}^{2}, x_{3}^{2}-x_{1} x_{2}$. By considering the coefficients of $x_{1} x_{3}$ and $x_{2} x_{3}$, we see that the given linear form must be, after multiplying by a constant in $k$, of the form $a x_{1}+b x_{2}+t x_{3}$ for some $t$ in $k$.

So we have to work out for which values of $t$ does the square $\left(a x_{1}+b x_{2}+t x_{3}\right)^{2}$ belong to our linear system of quadrics. We compute that, modulo this linear system:

$$
\begin{aligned}
\left(a x_{1}+b x_{2}+t x_{3}\right)^{2} & \equiv\left(a x_{1}+b x_{2}+t x_{3}\right)^{2}-(t / c)\left[\left(a x_{1}+b x_{2}+c x_{3}\right)^{2}-x_{1} x_{2}\right] \\
& \equiv\left(t^{2}-c t\right) x_{3}^{2}+(1 / c)(2 a b c-2 a b t+t) x_{1} x_{2} \\
& \equiv\left(t^{2}+(1 / c)\left(-c^{2}-2 a b+1\right) t+2 a b\right) x_{1} x_{2} .
\end{aligned}
$$

It is easy to check that $x_{1} x_{2}$ is not zero modulo our linear system of quadrics. So the square $\left(a x_{1}+b x_{2}+t x_{3}\right)^{2}$ belongs to our linear system of quadrics if and only $t$ satisfies the quadratic equation

$$
t^{2}+(1 / c)\left(-c^{2}-2 a b+1\right) t+2 a b=0 .
$$

Thus, the given linear system of quadrics contains the squares of two linear forms $x_{1}^{2}$ and $x_{2}^{2}$ defined over $\mathbf{Q}$, together with two others defined over the quadratic extension of $\mathbf{Q}$ corresponding to the above equation. It follows that this quadratic extension of $\mathbf{Q}$ is an invariant of the rational cohomology ring of the given 9 -manifold $Y$. It remains to show that as the rational numbers 
$a, b, c$ vary, we obtain infinitely many different quadratic extensions of $\mathbf{Q}$, and therefore infinitely many isomorphism classes of rational cohomology rings for these 9-manifolds.

The quadratic extension of $\mathbf{Q}$ given by the above quadratic equation is specified by the class of its discriminant $\Delta=B^{2}-4 A C$ in $\mathbf{Q}^{*} /\left(\mathbf{Q}^{*}\right)^{2}$. Here we have

$$
\Delta=4\left[\left(\frac{2 a b-c^{2}-1}{2 c}\right)^{2}-1\right]
$$

We assume that $\Delta$ is nonzero, as is clearly true for most $a, b, c$. Since $a, b, c$ can be arbitrary nonzero rational numbers, it is easy to see that the class of $\Delta$ in $\mathbf{Q}^{*} /\left(\mathbf{Q}^{*}\right)^{2}$ can be any element of the form $4\left(x^{2}-1\right) \sim x^{2}-1$ for $x \in \mathbf{Q}, x \neq \pm 1$. In particular, $\Delta$ can take infinitely many values in $\mathbf{Q}^{*} /\left(\mathbf{Q}^{*}\right)^{2}$ : for example, for any odd prime $p$, we can take $x=p+1$, and then $x^{2}-1$ has nonzero image under the homomorphism $\operatorname{ord}_{p}: \mathbf{Q}^{*} /\left(\mathbf{Q}^{*}\right)^{2} \rightarrow \mathbf{Z} / 2$. Therefore, the 9-manifolds we consider have infinitely many isomorphism classes of rational cohomology rings.

\section{Some possible substitutes for Grove's question}

The following questions can be viewed as substitutes for Grove's question. They are only slight extensions of well-known conjectures.

Bott's conjecture that simply connected manifolds with nonnegative curvature are elliptic ([8], p. 519) suggests that there should be strong restrictions on the homotopy type of manifolds with curvature $\geq-1$ and diameter $\leq D$. On the other hand, any conjecture must be compatible with Grove and Ziller's examples of nonnegatively curved manifolds, including all $S^{2}$-bundles over $S^{4}$ and all $S^{3}$-bundles over $S^{4}$ ([12], Theorem B). Any conjecture must also cover the almost nonnegatively curved manifolds found by Fukaya and Yamaguchi ([9], Theorem 0.18) and Schwachhöfer and Tuschmann [17], Theorem 4.2. By definition, a manifold $M$ has almost nonnegative curvature if for every $\epsilon>0, M$ has a Riemannian metric with $K_{M} \cdot \operatorname{diam}(M)^{2}>-\epsilon$. For example, any (linear) sphere bundle over a sphere has almost nonnegative curvature, by Fukaya and Yamaguchi.

Question 1. Is every closed simply connected manifold with nonnegative sectional curvature pure elliptic?

By definition, a manifold $M$ is pure elliptic if there is a minimal model for the cochain algebra $C^{*}(M, \mathbf{Q})$ such that the space of algebra generators $V=$ $V_{\text {ev }} \oplus V_{\text {odd }}$ is finite-dimensional (this says that $M$ is elliptic), while $d\left(V_{\text {ev }}\right)=0$ and $d\left(V_{\text {odd }}\right)$ is contained in the subalgebra generated by $V_{\mathrm{ev}}([8]$, p. 435). For example, every biquotient manifold is pure elliptic [13].

Question 2. Is every closed simply connected manifold with almost nonnegative curvature elliptic? 
An almost nonnegatively curved manifold need not be pure elliptic. For example, an $S^{5}$-bundle over $S^{3} \times S^{3}$ with nonzero Euler class has almost nonnegative curvature by Fukaya and Yamaguchi, but it is not pure elliptic. It is helpful to observe that for a pure elliptic space $M$, the Lie algebra $\pi_{\text {ev }}(\Omega M) \otimes \mathbf{Q}$ is abelian, while for $M$ elliptic, it is only nilpotent. Thus Questions 1 and 2 can be viewed as higher-dimensional analogues of the known results that a manifold with nonnegative curvature has almost abelian fundamental group [3], whereas a manifold with almost nonnegative curvature only has almost nilpotent fundamental group [9].

Question 3. Given $n$ and $D$, is there a finite set of closed Riemannian orbifolds $B_{i}$ such that every simply connected closed Riemannian $n$-manifold with sectional curvature $\geq-1$ and diameter $\leq D$ fibers over some $B_{i}$ with fiber almost nonnegatively curved?

Here "orbifolds" are allowed to have stabilizer groups equal to any compact Lie groups, not just finite groups. Question 3 is strongly suggested by Yamaguchi's Main Theorem (p. 318) and Conjecture (p. 323) [22]. Questions 2 and 3 together would imply that simply connected $n$-manifolds with curvature $\geq-1$ and diameter $\leq D$ have only finitely many sequences of rational homotopy groups $\pi_{*}(M) \otimes \mathbf{Q}$, using Friedlander and Halperin's determination of the possible rational homotopy groups of elliptic spaces ([8], p. 441).

\section{Acknowledgements}

I would like to thank Gabriel Paternain, Xiaochun Rong, and Krishnan Shankar for their papers, as well as many useful conversations.

\section{References}

[1] A. Adler, S. Ramanan. Moduli of abelian varieties, Lecture Notes in Mathematics, 1644. Springer-Verlag, Berlin, 1996.

[2] S. Aloff, N. Wallach, An infinite family of 7-manifolds admitting positively curved Riemannian structures, Bull. Amer. Math. Soc. 81 (1975), 93-97.

[3] J. Cheeger, D. Gromoll, On the structure of complete manifolds of nonnegative curvature, Ann. of Math. (2) 96 (1972), 413-443.

[4] A. Dold, Erzeugende der Thomschen Algebra R, Math. Z. 65 (1956), 25-35.

[5] J.-H. Eschenburg, New examples of manifolds with strictly positive curvature, Invent. Math. 66 (1982), 469-480.

[6] F. Fang, X. Rong, Fixed point free circle actions and finiteness theorems, Comm. Contemp. Math. 2 (2000), 75-86.

[7] _ Curvature, diameter, homotopy groups, and cohomology rings, Duke Math. J. 107 (2001), 135-158.

[8] Y. Félix, S. Halperin, J.-C. Thomas, Rational homotopy theory, Graduate Texts in Mathematics, 205. Springer-Verlag, New York, 2001.

[9] K. Fukaya, T. Yamaguchi, The fundamental groups of almost non-negatively curved manifolds, Ann. of Math. (2) 136 (1992), 253-333.

[10] M. Gromov, Curvature, diameter and Betti numbers, Comment. Math. Helv. 56 (1981), 179-195. 
[11] K. Grove, Critical point theory for distance functions, Differential geometry: Riemannian geometry (Los Angeles, 1990), 357-385. Proc. Symp. Pure Math. 54, Part 3, AMS, Providence, RI, 1993.

[12] K. Grove, W. Ziller, Curvature and symmetry of Milnor spheres, Ann. of Math. (2) 152 (2000), 331-367.

[13] V. Kapovitch, A note on rational homotopy of biquotients, preprint, 2002.

[14] B. O'Neill, The fundamental equations of a submersion, Michigan Math. J. 13 (1966), 459-469.

[15] G. Paternain, J. Petean, Minimal entropy and collapsing with curvature bounded from below, Invent. Math. 151 (2003), 415-450.

[16] A. Petrunin, W. Tuschmann, Diffeomorphism finiteness, positive pinching, and second homotopy, Geom. Funct. Anal. 9 (1999), 736-774.

[17] L. Schwachhöfer, W. Tuschmann, Metrics of positive Ricci curvature on quotient spaces, preprint: arXiv:math.DG/0303092

[18] D. Sullivan, Infinitesimal computations in topology, Inst. Hautes Études Sci. Publ. Math. 47 (1977), 269-331 (1978).

[19] W. Tuschmann, Geometric diffeomorphism finiteness in low dimensions and homotopy group finiteness, Math. Ann. 322 (2002), 413-420.

[20] C. T. C. Wall, Classification problems in differential topology. V. On certain 6-manifolds, Invent. Math. 1 (1966), 355-374.

[21] Nets of conics, Math. Proc. Camb. Phil. Soc. 81 (1977), 351-364.

[22] T. Yamaguchi, Collapsing and pinching under a lower curvature bound, Ann. of Math. (2) 133 (1991), 317-357.

DPMMS, Wilberforce Road, Cambridge CB3 0WB, England.

E-mail address: b.totaro@dpmms.cam.ac.uk 